\title{
Gestión de los conflictos y mediación social en Italia
}

\author{
Pierluigi CONSORTI \\ Universidad de Pisa \\ consorti@ddp.unipi.it
}

Recibido: 04/06/2012

Aceptado: 13/11/2012

\begin{abstract}
Resumen
Una reciente reforma legislativa impone en Italia la mediación preventiva de los conflictos civiles con implicaciones evidentes a nivel social y cultural. El mediador civil es un nuevo profesional que se ocupa de estos conflictos relacionales, y que ayuda a ver el conflicto como un lugar de encuentro: saber escuchar e identificarse con las razones del otro. A través del diálogo se pueden superar ritualidades contrapuestas e introducir procedimientos y soluciones alternativas de los conflictos modificando la tradicional contraposición error/razón y facilitando la comunicación. Esto está basado en el enfoque anglosajón del Alternative Dispute Resolution (ADR) y tal vez mucho más en una perspectiva gandhiana. Se propone, con respecto a este tema, una experiencia de mediación social desarrollada por el Centro Interdisciplinar de "Ciencias para la paz" de la Universidad de Pisa sobre la acogida de las personas sin hogar. Las actividades implementadas identifican la necesidad de trabajar sobre el contexto social y cultural de los ciudadanos (además de las políticas sociales) en una visión sistémica y holística que mire a la reconstrucción de las relaciones sociales de las personas sin hogar (recuperando sus rostros y sus historias individuales) con las personas que habitan a través de los caminos de mediación social. La finalidad es la de asumir un enfoque de cultural empowerment de las comunidades que pueda delinear nuevos modelos de convivencia incluyentes y de integración superando los temores estereotipados y los prejuicios sociales.
\end{abstract}

Palabras clave: la mediación legal, el conflicto social, cultural empoderamiento

\section{Conflicts Management and Social Mediation in Italy}

\begin{abstract}
Italian law recently imposed compulsory mediation before reaching a judge. That change produces important consequences at social and cultural level. A new professional profile arises, as a civil mediator, who address conflict by developing communicative process between parties in conflict. His/her aim is to create conditions for a paradigm shift from the traditional contraposition of conflicting parties through communication and comprehension of opposing views and understanding. Mediation involves the adaptation of a number of approaches to conflict including the Alternative Dispute Resolution (ADR) methodology or the Ghandian approach. The Interdisciplinary Centre "Sciences for Peace" (CISP) at Pisa University experienced in this regard through its research in the area of service provision for the homeless. The CISP research suggests that a holistic and systematic approach to conflict resolution is required. Such an approach requires that the social, cultural and political contexts of conflicting parties must be examined if social relationships are to be constructed or developed. To evolve from stereotypical fear and social prejudices, intervention through social mediation will
\end{abstract}


develop cultural empowerment within communities of focus and thus reduce or resolve conflict situations.

Keywords: legal mediation, social conflict, cultural empowerment

\section{Referencia normalizada}

Consorti, P. (2013). “Gestión de los conflictos y mediación social en Italia”. Política y Sociedad, Vol.50 núm. 1: páginas. 99-111

Sumario: 1.La nueva mediación obligatoria. 2.Un mediador de nuevo tipo. 3.La gestión de los conflictos en el ámbito social. 4.Un caso de estudio: sin hogar, mediación y gestión del conflicto social

\section{La nueva mediación obligatoria}

Desde el 20 de marzo de 2011 en Italia es obligatorio recurrir a la mediación antes de acudir a un juez civil. La razón principal de esta novedad se debe a la voluntad de aligerar la maquinaria judicial, que necesita demasiados años para alcanzar una sentencia definitiva. La duración excesiva de los procesos frustra de hecho la justicia civil ${ }^{1}$ : por eso la Unión Europea ha programado una disposición específica que ha condicionado el espacio jurídico del continente (Trocker, De Luca, 2011). La necesidad de abreviar los tiempos de los procesos ha producido un resultado paradójico que probablemente no ha sido todavía estudiado en profundidad (Carrara, 2011: 145). De hecho, la idea de la mediación obligatoria no es siempre convincente. Si tiene sentido mediar entre personas que desean llegar a un acuerdo, puede no tenerlo obligar a alguien a ponerse de acuerdo con su antagonista. Sobre todo si están en juego los derechos, a los que difícilmente estamos dispuestos a renunciar sin luchar.

No hemos llegado al Alternative Dispute Resolution (ADR) de origen americano $\mathrm{y}$, sin embargo, hemos dado un significativo paso adelante hacia la construcción de un sistema de mediación que, aunque no es todavía una alternativa, es por lo menos complementario con respecto a las formas de justicia ordinaria. El ordenamiento ya contemplaba otras formas de mediación en sectores particulares y muy limitados, por ejemplo para algunos debates agrarios o en temas de contratos laborales (Gabrielli, Luiso, 2005). La verdadera novedad del nuevo sistema se aprecia específicamente desde el perfil cultural, en cuanto estamos en definitiva llegando a desplazar la gestión del conflicto, que antes estaba sustancialmente solo en manos de los abogados, hacia una responsabilización de las partes, que están ahora

\footnotetext{
${ }^{1}$ Para la Corte Europea de los Derechos del Hombre la “duración razonable” de un proceso debe ser de cinco años entre el primer y el segundo grado: términos que en Italia casi nunca son respetados (Buffone, 2010: 6).
} 
obligadas a confiar en un mediador. No es tan extraño que precisamente los abogados hayan manifestado fuertes resistencias. Los profesionales tradicionales del conflicto no aceptan con mucho gusto que otros puedan sustituirlos en su trabajo.

Cometeríamos, sin embargo, un error si pensáramos este acontecimiento en términos de simples reivindicaciones sindicales. Como he dicho: el asunto es principalmente cultural, porque la obligación de intentar una conciliación antes de un proceso jurídico transforma los tradicionales criterios de gestión de conflictos. Para la cultura occidental, el proceso es el lugar típico del ejercicio de la defensa de los propios derechos, que se ponen en acción dirigiéndose al Estado en la figura del juez. El proceso transforma las personas en partes que, bajo el imperio de la ley, juegan un papel preciso. Uno se convierte en demandante, y el otro en demandado. Ambos deben ser asistidos por un letrado, o sea, un técnico que conoce bien las reglas del juego y por eso asume la defensa y en su nombre conduce el juego con el objetivo de ganar sobre la otra parte. De esta manera el proceso llega a ser parte del conflicto, y las razones del uno y del otro se esfuman hasta quedarse en el fondo de un escenario en el cual los técnicos acaban por ser protagonistas que se enfrentan para dirimir la causa. Los hechos se transforman en caso, las personas en partes, las soluciones en sentencias.

La reforma del 2010 es, sin embargo, solo la última de las tentativas de transferir la gestión del conflicto desde las salas de los tribunales hacia lugares distintos, siguiendo la lógica del ADR anglosajón (Nicosia, 2004) y, en los límites de lo posible, sustituyendo ritualidades contrapuestas con procedimientos cooperativos. La resistencia con la cual estos últimos son acogidos aclara una larga desconfianza popular hacia la oportunidad de considerar en positivo la idea que se pueda superar un conflicto sin una victoria. La idea cultural del conflicto que caracteriza la mentalidad común no se corresponde en realidad con los procedimientos de mediación.

Nos pasa a nosotros lo que se narra en un cuento judío. Dos personas en disputa deciden recurrir a un juez sabio. Se presentan, y el primero cuenta su versión de los hechos. El juez escucha atentamente, hace alguna pregunta y al final exclama: « ¡Tienes razón!». Entonces interviene el otro. Él también cuenta su versión de los hechos. El juez escucha con la misma atención; hace alguna pregunta y al final exclama: « $i$ Tienes razón!». En este momento uno de los presentes interviene: « ¡Excelencia - grita - no es posible que ambos tengan razón!». El juez lo mira, asiente con la cabeza y solemnemente confirma « ¡Tú también tienes razón!».

Esta historia expresa bien nuestra posición sobre la gestión de conflictos. A veces nosotros somos una de las partes integrantes que pide tener la razón; es más, casi siempre somos la persona que grita: «iNo es posible que ambos tengan la razón!». Si uno tiene razón, el otro tiene que estar equivocado. Un juez que hace bien su profesión debe dar la razón a uno y culpar al otro, si no, ¿qué juez es? Nuestra mentalidad cultural, y de manera particular aquella de los juristas, no está acostumbrada al arte de la mediación. Como máximo puede concebirse una transacción o un compromiso, pero difícilmente admitimos que puedan lograrse soluciones cooperativas en un conflicto. 
La cuestión es verdaderamente muy sugestiva si pensamos, por ejemplo, en las implicaciones de tipo neurológico que sostienen el actuar humano. Ya conocemos mucho más sobre «cómo funcionamos» y estamos preparados para advertir las conexiones que relacionan nuestras acciones, nuestra manera de ser - en una palabra, nuestro comportamiento -con modelos que las neurociencias son capaces de explicar, si no mejor, por lo menos igual que las ciencias cognitivas. Bajo esta perspectiva, creo que se necesita todavía reflexionar para comprender que el éxito de las formas de gestión conciliativa de los conflictos no se medirá tanto en su capacidad de deflación del contencioso, cuanto sobre la eficacia de estas prácticas en términos de inteligencia emocional. Es decir, admitiendo, como decía Manzoni, que «el mal y la razón no se cortan nunca con el cuchillo» (Picozza, 2011: 9); o en términos más actuales, mostrando confianza en creer que los conflictos puedan ser gestionados y finalmente transformados, y no solo solucionados en términos de la victoria de una parte sobre la otra.

La necesidad de recurrir obligatoriamente a la mediación antes de demandar - en sentido estricto y tradicional - por «vías legales», obligará a cambiar costumbres consolidadas en el tiempo. Si hasta ahora sabíamos que para resolver una controversia teníamos que «ir al abogado», ahora tendremos que aprender a ir al mediador. Un cambio práctico que producirá formidables consecuencias culturales.

\section{Un mediador de nuevo tipo}

En la nueva ley el mediador es el profesional de los conflictos relacionales (Nicosia, 2011). Para entender mejor esta observación es necesario dar un paso atrás y precisar que, a diferencia de lo que se piensa a menudo - especialmente entre quien no conoce el tema de la gestión de conflictos - el conflicto no constituye necesariamente un elemento patológico de la relación personal y/o social. Es necesario convencerse de que el conflicto es un elemento fisiológico, por lo tanto natural, de la vida. No tiene sentido enfrentarlo con una actitud hostil. Y más en general, antes que enfrentarlo estudiando las estrategias para vencer, es mejor profundizar en las maneras para convencer (Arielli, Scotto, 2003). Es decir que no debemos secundar la mentalidad común que nos pone delante del conflicto como si estuviésemos en un ring de boxeo. Cuando estamos dentro de un conflicto demasiadas veces ingeniamos las maneras para destruir al otro. Negamos cada una de sus razones - incluso más allá de la sensatez - porque pensamos que así venceremos (Hampshire, 1989). Lo último que nos parece razonable es cooperar con el otro, precisamente porque es nuestro adversario. Mark Juergensmeyer (1984) que aplica el método gandhiano para la solución de los conflictos, cuenta una historia común, que en términos jurídicos integra el caso de la «oposición de términos», y describe la gestión gandhiana. Dos propiedades están divididas por un patio común. En los mapas la línea de fronteras es evidente, pero en realidad no se entiende bien por dónde pasa. Los dueños saben que ésta empieza en cierto árbol y sigue a lo largo de una cierta dirección identificada con una antena sobre una colina. Pero, en la 
práctica, hay incertidumbre sobre el punto exacto de división del patio: y esto provoca la disputa. Uno acusa al otro de que los nietos juegan a la pelota en su lado, o que el automóvil ha sido aparcado en un lugar equivocado. Que esa planta no ha sido plantada precisamente a lo largo del límite, sino un poco más allá. Moverla no sirve, habría que cortarla. Los dos dueños no se sienten bien con el conflicto. La única solución parece ser la de acudir al abogado, y después al juez, para que él establezca en definitiva quién tiene razón y quién no. Sin embargo, un día llega a este lugar un buen mediador. Le cuentan sus problemas despotricando el uno contra el otro a causa de la pelota, del automóvil aparcado y de la planta inoportuna. El mediador se interesa por la cuestión y empieza a hablar con ellos. Hace algunas preguntas y entiende que las razones del conflicto son mucho más profundas. La definición de los derechos relacionados con la propiedad esconde solo un montón de antiguas y muy profundas rivalidades que caracterizan la historia de las dos familias. Cosas nunca dichas a las que hacen referencia ciertas calumnias del pueblo. Todo parece tener su origen en la supuesta «dudosa moralidad» de una de las hijas de A que, no obstante, es muy amiga de la hija de B. Además, se descubre que también las mujeres son muy amigas, y se ayudan regularmente en la educación de los hijos. El caso es que A y B, quienes pasan mucho tiempo fuera de casa, no sabían de esas relaciones. Empezando a hablar, los dos reconocen tener en común la pasión por la pesca. Así, mientras hablan y al final se encuentran, su atención se desplaza de los elementos que caracterizaban el conflicto que los convocaba, para extenderse en un contexto más vasto: dentro del cual el conflicto tomaba espacio. El mediador ha facilitado, pues, la comunicación entre los dos vecinos, haciéndoles caer en la cuenta de que tenían en común mucho más que el patio. Pero ellos no lo habían imaginado nunca; no se lo habían dicho nunca. Su problema no es tanto la pelota, sino el ruido que en ciertas horas de la tarde impide descansar. Además, sería suficiente mover el automóvil un par de metros a la derecha para sacar el espacio suficiente para aparcar también un segundo coche: la solución estaba delante de los ojos, pero se necesitaba desnudar el conflicto de los elementos de principio para considerarlos sobre los intereses de cada uno. Y también la planta destinada a ser cortada, al final continúa en pie: ha sido suficiente ponerse de acuerdo en la poda y en una limpieza más frecuente de las hojas que caen al suelo.

Este relato, que no es una fábula, nos ayuda a analizar la filosofía que presupone la nueva legislación italiana, que atribuye a un nuevo profesional la tarea de atender las partes en conflicto para que hallen una solución sin recurrir necesariamente al juez.

En realidad, la ley atribuye a los mediadores una tarea más incisiva. No deben solo ayudar a las partes a hallar un acuerdo amistoso (hasta aquí, nada de nuevo), sino que pueden también formular una propuesta de mediación. Es decir, pueden intervenir en el conflicto evaluándolo y ofreciendo una hipótesis de solución: algo que se parece más a una decisión de autoridad que a una facilitación. Los mediadores profesionales que se han formado en las escuelas del arte de la mediación no podrían hacer nunca una cosa de este tipo. El imperativo de la imparcialidad los obliga a estimular la búsqueda de un acuerdo, que debe surgir de las partes. Cuidan 
de poner en evidencia las respectivas razones haciéndolas encontrarse con sus intereses, pero sin expresar una evaluación sobre el conflicto y mucho menos sobre cómo las partes actúan dentro del conflicto. El mediador madura evidentemente una idea de la cuestión, pero no debe hacer presión sobre las partes en la dirección en que su opinión sería mejor. Justamente la ausencia de una autoridad evaluadora distingue al mediador del juez: las partes saben que no serán juzgadas por el mediador, sino solo ayudadas para que puedan hacerse una mejor idea sobre cómo están las cosas para poder alcanzar un acuerdo consensual (Valdambrini, 2009).

La exigencia de desincentivar el recurso al proceso civil ha llevado al legislador italiano a realizar una elección criticable desde el punto de vista del mediador profesional. Obviamente él no está obligado a aprovechar esta oportunidad de decidir. Puede y no debe formular una propuesta conciliadora (Ristori, 2011: 84). Aunque ningún mediador profesional hiciera una cosa parecida, es posible que algún mediador prefiera ponerse en el lugar del juez, por lo tanto valorar los hechos y proponer su solución. Cosa que la ley mira con una cierta simpatía: porque si las partes no aceptan tal propuesta y después el juez debiera decidir sobre el mismo asunto, la parte "que vence” tendrá que pagar una fortísima sanción económica por haber obligado a la justicia a intervenir en una cuestión que hubiera sido ya resuelta en una mediación.

También bajo este punto de vista la voluntad de aplazar el proceso se ha enfrentado por tanto con aquel déficit cultural que hemos indicado. El punto fuerte de la nueva ley es al mismo tiempo su talón de Aquiles: la obligatoriedad de la mediación puede parecerse a un boomerang. La obligación de recurrir a la mediación antes del proceso civil hará usual la práctica mediadora, que - al menos, al comienzo - será vivida con los viejos criterios culturales. Personas acostumbradas a vivir el conflicto de manera contrapuesta y según la lógica ganar/perder - basada sobre la buenas razones que justifican los derechos de unos contra los deberes de otros - se sentirán obligadas al acuerdo y por lo tanto lo evitarán. No colaborarán en el intento de conciliación porque de hecho no es posible obligar a alguien a sentarse al lado de su adversario, para encontrar forzosamente un acuerdo. Esto es posible entre amigos que ya han decidido hallar una solución común a un problema común, pero no somos todavía capaces de razonar sobre nuestros derechos poniéndolos a disposición dentro de una relación conflictiva, lo que parece contradecir una lógica cooperativa.

El desafío está de todas maneras abierto. Basta tener claro que no jugamos solo una partida contra la ineficacia de la justicia, sino una competición más amplia y profunda, de tipo cultural, que concierne la dimensión misma de la conflictividad y nuestra manera de ponernos en relación con ella. Ya no es hacer que se respeten nuestros derechos y nuestras razones contra las de los otros, sino ponerlas en relación con las de los otros (Consorti, 2009: 13). 


\section{La gestión de los conflictos en el ámbito social}

Ahora es útil considerar la oportunidad de diferenciar entre gestión y solución del conflicto. La cuestión ha sido ya tratada en la literatura (Arielli, Scotto, 2003) y no es oportuno hacer un recorrido de los resultados. Es suficiente aclarar los términos generales del tema, que podemos simplificar distinguiendo entre aquellos que, imaginando el conflicto como un elemento patológico de la vida, tienen como objetivo evitarlo, o de todas maneras, superarlo. Quien piensa en el conflicto como un nudo que desatar, obviamente querrá in primis prevenirlo y resolverlo (Mitchell, Banks, 1996).

Por otra parte, existen quienes pueden mirar el conflicto como un elemento fisiológico de la vida. Algo natural que no tiene que ser por lo tanto necesariamente evitado. Más bien, puede ser valorado; puede entenderse su lado humano ${ }^{2}$, puede analizarse (Tilly, Tarrow, 2008), puede incluso aprenderse a gestionarlo ${ }^{3}$.

Manejar un conflicto significa en esencia transformarlo. O sea, privarlo de la carga destructiva que potencialmente le pertenece cuando se afronta con las herramientas tradicionales de la lógica «ganar o perder» ${ }^{4}$. El ya mencionado enfoque gandhiano en la gestión de los conflictos demuestra cómo estos se deben a la diferencia entre puntos de vista. Cada uno ve las cosas a su manera: una manera que parece la correcta. Por lo cual parece inútil obligar a encontrar un acuerdo si antes no se consigue establecer una relación entre los puntos de vista, logrando que se comuniquen las personas que los sostienen ${ }^{5}$.

${ }^{2}$ Este es un enfoque en principio típicamente originario de la psiquiatría (Fornari, 1969; Mitscherlich, 1972; Nivoli, 2003), después retomado también en el sentido político e internacionalista (Toscano, 2000; Howard, 2002).

${ }^{3}$ No uso la expresión «gestión» en el sentido que le atribuye Scotto (2003: 161) donde señala - sobre la base de los estudios del economista austriaco Friedrich Glasl - los «aspectos de la previsibilidad y capacidad de gestión del fenomeno conflictual» que le darían una connotación eficientista que implica «la necesidad de hacer gestionar los efectos destructivos del conflicto por un especialista». Me permito no valerme de los cánones epistemológicos de la sociología de los conflictos, por lo tanto atribuyo a esta expresión un sentido más amplio, incluido el elemento de transformación de los conflictos (al cual sin embargo no atribuyo todas la consecuencias técnicas que considera Scotto, 2003: 162 ss.), y que son muy amadas por los estudiosos de J. Galtung (v. Salio, 2009).

${ }^{4}$ Véanse las obras de Johan Galtung, considerado por algunos el fundador a nivel académico de la Peace Research (Altieri, 2008: 7). Confieso que en los escritos de Galtung no consigo encontrar inspiraciones significativas para merecer tal título, aunque reconozco que he sabido obtener ventaja útil de su formación matemática para construir modelos de interpretación de los conflictos, que le han permitido ejercer actividades de mediación y de capitalizar su experiencia, dando vida a distintas asociaciones y centros de investigación que ponen en práctica su método.

${ }^{5}$ «Se trata, más bien, de salir de la lógica del error y de la razón para acceder a la lógica de la comunicación» (Cozzo, 2004: 78). 
Podemos a estas alturas preguntarnos por qué alguien convencido de sus buenas razones debería ceder a las razones del otro, sin estar tampoco obligado. Hay varias respuestas, todas opinables (depende al final de los puntos de vista). Podremos justificar el cansancio de estar dentro un conflicto; o la incapacidad de ver una solución clara de manera razonable; o suponer que mientras tanto han cambiados los motivos que sostenían una cierta posición. Podemos también pensar que la solución de una victoria impuesta no es realmente satisfactoria (sucede que la decisión de un juez a menudo, aunque conforme a la ley, desatiende los elementos de verdad que de todas maneras existen en la posición del perdedor) y también que alcanzar un compromiso deja a las partes insatisfechas (un poco perdedoras y un poco victoriosas). La razón verdadera, sin embargo, consiste en darse cuenta de que ninguno tiene toda la razón y ninguno está totalmente equivocado. Transformar el conflicto significa esencialmente hacerlo un sitio de encuentro en lugar de uno de conflicto. Manejar el conflicto significa saber escuchar e identificarse con las razones del otro. Significa admitir que el otro no es necesariamente un enemigo (GossMayr, 1997). Conducir un conflicto según estas líneas construye relaciones verdaderas que superan el conflicto mismo y producen inesperadas dinámicas creativas ${ }^{6}$.

Quien se limita a observar el conflicto para proponer una solución convincente partiendo de sus propios criterios de comprensión, cae fácilmente en el cliché ganar/perder, error/razón. Termina así tomando casi exclusivamente decisiones tranchante porque está convencido de que no es posible que ambos tengan razón.

Haciendo esto se comete un error muy grave. En primer lugar, porque se sale de la lógica de la gestión del conflicto para adoptar aquella de la (supuesta - como hemos dicho - ) solución; y, sobre todo, porque se da inicio a un innumerable tipo de conflictos que no son sino consecuencia de la incomunicabilidad inicial y la incomprensión de las respectivas razones.

\section{Un caso de estudio: sin hogar, mediación y gestión del conflicto social}

El caso de estudio concierne a los conflictos sociales. Me inspiro en una actividad desarrollada por el Centro Interdisciplinario de Ciencias por la paz de la Universidad de Pisa con motivo de la apertura de un albergue para personas sin hogar. Se trata de una situación ya ordinaria en muchas ciudades europeas, codificada como "síndrome Nimby". Ocurre también en relación con la apertura de campos de nómadas o refugiados. La comunidad que vive cerca de las zonas escogidas por la administración rechaza la elección del lugar, buscando mil buenas razones para explicar que es poco adecuado. El lugar idóneo es siempre un poco más allá. Muy a menudo la discusión asume tonos violentos. Por eso la administración pública

\footnotetext{
${ }^{6}$ Sobre la cuestión de la diferencia entre método y reglas conductuales en la lógica resolutiva del conflicto, cfr. Drago, 1997: 237 - 250.
} 
tiende a no advertir a la población, situándola frente al hecho consumado. Una opción poco prudente, que en realidad a veces desencadena verdaderos tumultos públicos. En estos casos es siempre muy difícil dar cuenta de las razones; así prevalecen los prejuicios y la mediación da espacio a la discusión entre ciudadanos y las fuerzas de policía que deben mantener el orden público.

Desde nuestro punto de vista es interesante preguntarnos por qué nos sentimos en conflicto con las personas sin hogar (pero la lógica es la misma si pensamos en los extranjeros irregulares o en los refugiados). Por qué ante la hipótesis descrita elegimos que la finalidad predominante de la supuesta armonía social, que sería desestabilizada por la presencia de un albergue de los sin hogar, justifica una actuación a veces contradictoria, que puede llegar hasta la utilización de medios violentos. Con la finalidad de mantener la paz de partida se sacrifica hasta la paz misma; así, el conflicto se presenta como el límite estructural de la paz.

No es este el lugar para discutir en términos exhaustivos el problema propuesto (Consorti, 2011). Deseo solamente poner en evidencia que el punto de partida, o sea, la necesidad de dar hospedaje a los sin hogar, constituye el casus belli de la dinámica conflictual que examinamos. Parece que la tradicional respuesta social: dar hospedaje a quien no lo tiene, fuera la única respuesta posible. Por lo tanto cada espacio de mediación sobre una hipótesis distinta resultaría frustrado a priori.

Encontramos aquí un doble límite: social y estructural. Desde el primer punto de vista (social), deberíamos preguntarnos si ser "sin" significa de por sí exclusión y no constituye en cambio una forma de inclusión. Los "sin" tienen en realidad un lugar bien preciso en el contexto social. Viven al margen; y precisamente su posición marginal asegura que los otros estén en posición central. Quien "tiene” está en una posición central justo por el hecho de no estar "sin" algo. La presencia marginal de los "sin" asegura la posición central de quien tiene, legitimando la tendencia a mantener a los otros - los "sin" - al margen. En esencia, se perpetúa un esquema que después de todo sirve para el mantenimiento de un sistema social que, preocupándose de asegurar ciertas respuestas básicas sin invertir las posiciones, confirma su validez global(White L., 2000). Del segundo punto de vista (estructural) podemos constatar que existe una dificultad concreta en el dar a los "sin" lo que les falta. Confiar en una respuesta de bienes o servicios es necesario, pero al mismo tiempo no es suficiente. Y también la respuesta más refinada, que consiste en el imaginar servicios o "redes" que enseñen a recuperar el espacio vacío del "sin", se enfrenta con una realidad que supone que lo que falta existe, y antes o después se puede conseguir. ¿Pero es siempre verdad?

Formulo preguntas sin respuestas, que no obstante demuestran la complejidad y pueden ayudar a encuadrar científicamente el problema situándolo en una lógica distinta de la tradicional, que hace después de todo tranquilizadora la presencia de las personas sin hogar, en la medida en que no me concierne; hasta que no llega a ser un problema mío. En esta eventualidad salta el miedo; y las personas sin hogar se perciben como enemigos. Me parece importante subrayar que en casos similares a éste emergen temores estereotipados a menudo, unidos a una representación típica de la pobreza. El vagabundo, que constituye el cliché de la persona sin hogar, asusta 
en la medida en que representa este cliché de pobreza y de "desadaptación social” sin escape. No sentimos ningún temor hacia una persona sin hogar si la encontramos en un tren bien vestida y por tanto no la reconocemos como el "otro sin hogar".

Además, hay que observar el formidable peso que en casos similares asume la expectativa de violencia, antes aún que la violencia misma. Presten atención: cuando se trata con los pobres, el miedo de la posible violencia juega un papel más significativo del que tiene lugar en otros conflictos explícitamente y típicamente violentos. No todas las veces que entramos en un banco pensamos en un posible robo; en cambio todas las veces que encontramos un pobre tememos la agresión.

En general la mediación de los conflictos sociales funciona respecto de conflictos comprobados y en presencia de violencias específicas o identificables (un piquete, una barricada inicial...), en cambio, cuando se habla de las personas sin hogar la sola expectativa de violencia posible genera un conflicto que produce contraposiciones reales también en ausencia de una efectiva y razonable causa de conflicto. El "principio de precaución" provoca mecanismos conflictivos y estrategias unilaterales de defensa antes que el conflicto se haga manifiesto como tal (Sunstein, 2010). Aparece el miedo, y el "conflicto virtual" se convierte inmediatamente en real; produce distancias y separaciones efectivas que no pueden aproximarse con las dinámicas de mediación clásicas. Aquí hay un primer punto de atención. Si por un lado es verdad que "no soy racista, pero ..." (por lo cual considero justo acoger a los refugiados, siempre que sea en otro lugar; o también dar casa a quien tiene necesidad, pero no a los gitanos), por otro lado, observamos que cuando desestructuramos el imaginario del "pobre-otro", del "vagabundo-peligroso" las relaciones, incluidas aquellas violentas, pueden cambiar.

Esto significa que es posible intervenir sobre la carga conflictiva ligada a la condición de las personas sin hogar, mejorando su misma condición social, trabajando más sobre el contexto y no necesariamente sobre ellos.

Un segundo punto de vista podemos asumirlo leyendo la relación entre personas sin hogar y conflicto social en una visión sistémica, donde la homelessness representa un "nudo" del tejido social en el cual se enredan cuestiones externas (refugiados, migrantes...) y cuestiones internas (políticas sociales, forma de ciudadanía, marginación social ...).

El conflicto social, para retomar el leitmotiv inicial, constituye un estado fisiológico de las relaciones sociales incluidas dentro de este esquema. La desigualdad social, así como la difícil convivencia entre los diferentes, produce situaciones marginales que en la imagen sistémica vuelve a proponer la cuestión de las personas sin hogar como uno de los "nudos" más periféricos del territorio. El concepto de "alta marginalidad" representa la imagen del límite de las acciones sociales y termina por ser un problema demasiado grande para que se resuelva de verdad. Las personas sin hogar se encuentran ya en una condición de no retorno, destinados a quedarse para siempre al margen de la sociedad. Lo máximo que se puede hacer por ellos al final coincide con lo mínimo: darles un hogar y, si tampoco lo quieren (como pasa), darles asistencia primaria. 
Si depuramos los cliché - también académicos - y recuperamos los rostros de las personas sin hogar, nos damos cuenta que ellos expresan cada uno una historia. De esta manera cada uno emerge del "pueblo de los invisibles"; el encuentro con la marginalidad (que en algunos casos puede coincidir con el mismo nacimiento) vuelve a ser un dato histórico, un pedazo de sus vidas, y no un elemento del destino o del resultado del cálculo de las probabilidades.

En este sentido la relación entre personas sin hogar y conflicto social es doble: por una parte la homelessness es el resultado de conflictos (sociales, entendidos en un sentido amplio que comprende todas las políticas socio-económicas, pero no solo sociales, como el caso de los refugiados huidos de las guerras); por otra es productor de conflictos, por el solo hecho de que expresan historias de diversidad marginal.

En estos casos el proceso de mediación puede actuar por lo menos sobre dos niveles.

El primero concierne a la gestión del problema de los sin hogar. Con respecto a esto es necesario observar que la solución no es solo aquella de dar un techo. Se necesita reconstituir sus relaciones sociales, porque hay necesidad de un "hogar en sentido estricto", que incluya la construcción de redes sociales antes que la adjudicación de una vivienda (Lemos G., 2000).

El otro concierne a la gestión de los problemas sociales conectados a la presencia de los sin hogar. En otras palabras se necesita enfrentar el conflicto sistémico que surge ya sea de la presencia de los sin hogar, o de la presencia de formas de asistencia construidas en teoría sin la participación de las comunidades. Otra vez es necesario encuadrar la gestión de conflictos similares en el ámbito de la realización de caminos de mediación social, entendidos como herramienta de construcción (y reconstrucción) de las relaciones.

En contextos similares, la mediación ya no se entiende en el sentido técnico mencionado como premisa (intervención de un tercero neutral), sino en su significado más profundo: la facilitación de empowerment en la gestión de la conflictividad. Así, ésta se presenta como una alternativa a la lógica de la intervenciones de excepción (falta de vivienda, entonces ofrezco un hogar temporal) trabajando sobre la dimensión social global. Por otra parte, la dimensión del empowerment anuda entre ellos varias relaciones de la comunidad. Por un lado mueve recursos de abajo que pueden ser dirigidos hacia formas de acogida y solidaridad más eficaces que las respuestas institucionales (que proceden de arriba); por otro lado pueden asignar, en contra de lo que comúnmente se cree, también a las personas sin hogar el carácter de recursos sociales valorizando la presencia activa en las formas de compromiso social. En sentido propio, por ejemplo incluyéndolos con todo derecho en los procesos de decisión conectados al territorio en el cual viven, y de manera indirecta, por ejemplo obligando a los involucrados a asumir sus necesidades específicas como necesidades del territorio y no solo como "problemas suyos".

La valorización global de los recursos humanos, sin demasiada diferencia entre sin hogar y con hogar, demuestra además cómo a través de "recursos marginales" se pueden transformar los conflictos y desarrollar nuevos modelos de convivencia. Es 
más necesario programar intervenciones que hagan contemporáneamente presión sobre estrategias de promoción de los recursos individuales de los sujetos vulnerables y sobre las potencialidades incluyentes de las comunidades territoriales que se enfrentan con el fenómeno de la grave marginalidad social.

El trabajo de campo desarrollado en Pisa ha demostrado la posibilidad de integrar las intervenciones de asistencia primaria con el soporte de un sistema relacional. Donde ha sido posible ofrecer tales "bienes relacionales", resultado de interacciones constructivas con los habitantes del barrio, debidamente preparadas por los operadores sociales, se ha encontrado la puesta en marcha de un círculo virtuoso que ha visto a las personas sin hogar activarse de manera más adecuada dentro de sus propios caminos, al tiempo que la comunidad territorial ofrece espontáneamente respuestas útiles para facilitar la integración.

Tal enfoque une las dos dimensiones identificadas al comienzo, porque permite intervenir sobre el problema global de los sin hogar - es decir, sobre la dimensión relacional y de integración, pasando más allá de la dimensión material conectada a la necesidad de casa y comida - junto a los problemas que su presencia produce en los territorios.

Este enfoque holístico transforma el conflicto.

\section{BIBLIOGRAFÍA}

Altieri R. (2008), "Presentazione. Johan Galtung e le scienze per la pace”, in J. Galtung, Affrontare il conflitto. Trascendere e trasformare, Plus- Pisa University Press.

Arielli E., Scotto G. (2003), Conflitti e mediazione: introduzione ad una teoria generale, Mondadori.

Buffone G. (2010), "Risoluzione alternativa delle liti civili e commerciali. Mediazione e conciliazione delle controversie civili e commerciali disciplinate dal D. lgs. 4.03.2010, n. 18” Il civilista, 4: 5-34.

Carrara C. (2011), “Art. 7. Effetti sulla ragionevole durata del processo" La nuova disciplina della mediazione delle controversi civili e commerciali. Commentario al d. lgs. 4 marzo 2010, n. 18, Giuffré: 141-147.

Consorti P. (2009),"'Hanno ragione tutti!' Profili di gestione dei conflitti interculturali ed interreligiosi” Gestire i conflitti interculturali ed interreligiosi. Approcci a confronto (Consorti P., Valdambrini A., edd.), Plus - Pisa University Press: 9-30.

Consorti P. (2011), “Homelessness, Social Mediation and Peace”, Scienza\&Pace, novembre: 1-18. 
Cozzo A. (2004), Conflittualità nonviolenta. Filosofia e pratiche di lotta comunicativa, Mimesis.

Drago A. (1997), "La dinamica della soluzione nonviolenta dei conflitti: un modello suggerito dalla storia della scienza”, Peacekeeping e Peacebuilding. La difesa e la costruzione della pace con mezzi civili, (Drago A., ed.) Qualevita.

Fornari F. (1969), Dissacrazione della guerra: dal pacifismo alla scienza dei conflitti, Feltrinelli.

Gabrielli E., Luiso F.P. (2005), I contratti di composizione delle liti, Giappichelli.

Goss-Mayr H. (1997), Come i nemici diventano amici. Insieme per la nonviolenza, la giustizia e la riconciliazione, EMI.

Hampshire S., (1989), Innocenza ed esperienza. Un'etica del conflitto, Feltrinelli.

Howard M. (2002), L'invenzione della pace. Guerre e relazioni internazionali, Il Mulino.

Lemos G., (2000), Homelssness and Loneliness. The want of conviviality, Crisis.

Mitchell C.R., Banks M. (1996), Handbook of Conflict Resolution. The Analytical Problem-solving Approach, Pinter.

Mitscherlich A. (1972), L' idea di pace e l'aggressivita umana: quattro saggi, Sansoni.

Nicosia P.S. (2004), Come gestire i conflitti e vincere insieme, Paoline.

Nicosia P.S. (2011), "Media-conciliazione. Appunti sul decreto legislativo 28/2010" Scienza\&Pace, 2: 1.

Nivoli G.C. (2003), Uomini alla guerra. La patologia mentale del guerriero, Centro scientifico editore.

Picozza E. (2011), Neurodiritto. Un'introduzione, Giappichelli.

Ristori L. (2011), “Art. 5. Condizione di procedibilita e rapporti con il processo” La nuova disciplina della mediazione delle controversie civili e commerciali. Commentario al d. lgs. 4 marzo 2010, n. 18, Giuffré: 75-98.

Salio N. (2009), "La trasformazione non violenta dei conflitti secondo il metodo Transcend", Gestire i conflitti interculturali ed interreligiosi. Approcci a confronto (Consorti P., Valdambrini A., edd.), Plus - Pisa University Press: 115132.

Sunstein C.R. (2010), Il diritto della paura. Oltre il principio di precauzione, Il Mulino.

Tilly C., Tarrow S. (2008), La politica del conflitto, Mondadori.

Toscano R. (2000), Il volto del nemico. La sfida dell'etica nelle relazioni internazionali, Guerini e associati.

Trocker N., De Luca A. (2011), La mediazione civile alla luce della Direttiva 2008/52/CE, Firenze University Press.

Valdambrini A. (2009) "Una cornice teorica per la gestione e trasformazione dei conflitti interculturali ed interreligiosi", Gestire i conflitti interculturali ed interreligiosi. Approcci a confronto (Consorti P., Valdambrini A., edd.), Plus Pisa University Press: 159-184.

White L. (2000), "On Abolitionist Critiques, Homeless Service Programs, and Pragmatic Change”, St. Louis University Public Law Review: 431-445. 